\title{
Evaluation of Crude and Modified Cordia myxa Gum for its Nutraceutical Benefits
}

\author{
M. F. TAHIR, G. MUSTAFA, RAZIA NOREEN, JAVARIA ALTAF¹, FOZIA ANJUM² AND SHAZIA ANWER BUKHARI* \\ Department of Biochemistry, ${ }^{1}$ Department of Zoology, ${ }^{2}$ Department of Chemistry, Government College University, \\ Faisalabad 38000, Pakistan
}

Tahir et al.: Nutraceutical Benefits of Cordia myxa Gum

\begin{abstract}
Plants are a rich source of safe and valuable bioactive compounds and since ancient times they have been used as medicines. Medicinal herbs are used as traditional means of treating many human diseases in many parts of the world. In current study, the green synthesis pathway was investigated for modification, reducing and crown potential of Cordia myxa gum for the synthesis of silver nanoparticles. The seeding process and the synthesis of silver nanoparticles were tracked by taking ultraviolet/visible absorption spectra during the reaction. Nutritional as well as medicinal potential of raw and modified gums were analyzed. Synthesized spherical nanoparticles were up to $65 \mathrm{~nm}$ in size. The ultraviolet/visible spectroscopic results revealed an intense peak at $428 \mathrm{~nm}$ together with other small peaks in the region of 390-450 $\mathrm{nm}$ due to the presence of nanoparticles of polydispersed silver. The purified gum showed the highest phenolic contents (i.e. $14.71 \pm 0.04 \mathrm{mg}$ ) and percentage scavenging activity (i.e. 87.92 \pm 2.25 ). The maximum bactericidal activity was showed by nanoformulated gum against Escherichia coli with inhibition zone of $42 \mathrm{~mm}$ while minimum was found to be against Staphylococcus aureus with inhibition zone of $14 \mathrm{~mm}$. Nano formulated gum samples also exhibited optimal fungicidal effects against Fusarium solani with inhibition zone of $11.09 \mathrm{~mm}$ and Aspergillus niger with inhibition zone of $15.53 \mathrm{~mm}$. The results of current study portrayed that modifications in Cordia myxa gum would be beneficial to food products as an additive.
\end{abstract}

Key words: Cordia myxa, silver nanoparticles, phenolics, antimicrobial activities, gum based nanoformulation

Today, plant research is the center of attraction because it has the potential to be used in various areas of the world's industry. A wide range of natural materials is used to maintain the health of all living beings ${ }^{[1]}$. About $80 \%$ of the world's population still depends on traditional medicine. Cordia myxa (C. myxa), commonly known as 'lasura' belonging to family Braginaceae, possessed analgesic, antiinflammatory, immunomodulatory, antimicrobial, antiparasitic, insecticidal, cardiovascular, respiratory, gastrointestinal and protective effects while traditionally $C$. myxa is used in Ayurveda, Unani and Siddha systems of medicine ${ }^{[2]}$. Plant materials of C. myxa include various phytoconstituents including gum. Gums have been known since ancient times as plant products ${ }^{[3]}$. Gum C. myxa is obtained from its fruits and its modified forms are also used for the drug delivery system. Chemical and physical modifications are used to improve the functional properties of the gum as a biopolymer ${ }^{[4]}$.

*Address for correspondence

E-mail: shaziabukhari@gcuf.edu.pk

July-August 2021
Using silver nanoparticles (AgNPs) for different purposes has been gaining attention in recent years. AgNps formulated gums are progressively played large role in different disciplines such as food, health care, medical and industrial purposes because of their particular chemical and physical properties ${ }^{[5]}$. Nanoparticles can be synthesized using various biological, chemical and physical methods. Biological methods, on contrary to chemical methods, not only result in the productions of non-hazardous products but also are energy efficient. For that reason, chemical synthesis of nanoparticles is expensive ${ }^{[5]}$. As compared to other biological systems, biosynthesis of nanoparticles by means of plants and plant based

\footnotetext{
This is an open access article distributed under the terms of the Creative Commons Attribution-NonCommercial-ShareAlike 3.0 License, which allows others to remix, tweak, and build upon the work non-commercially, as long as the author is credited and the new creations are licensed under the identical terms
} 
extracts tends to be harmless, less time consuming and have a lesser farming cost. Moreover, plant based biosynthesis is a somewhat simple method that can be expanded to produce nanoparticles of large proportions ${ }^{[6]}$.

Gums being nontoxic, biologically active, biodegradable, biologically compatible and capable to modify chemically, more over economic and easily available have made their attraction towards pharmacological applications. Various research studies have revealed that gums have advantage over synthetic polymer or materials and can be used potentially or can lead fascinating discoveries in pharmaceutics and food industry. Therefore, the current study was designed to purify and modify C. myxa gum by acrylamide grafting. Bioanalytical techniques were employed to investigate and characterize natural and modified C. myxa gum for the synthesis of nanoformulations. The study will add more benefits of this modified/formulated gum to our foods and/or other edible formulations as functional additives in future.

\section{MATERIALS AND METHODS}

\section{Chemicals and reagents:}

The chemicals used were at least of analytical grades and of companies such as Sigma-Aldrich (U.S.A), Fluka (U.S.A), BB1 (UK), Oxoid (UK), Merck (Germany), Pharmacia and ICN.

\section{Target sample for research:}

Gum C. myxa was purchased commercially from herbal medicine store 'Bada Dawakhana' Karkhana Bazar, Faisalabad. The collected gum was solid and reddish brown in color. Gum sample was subjected to identification and authentication by Department of Botany, Government College University, Faisalabad, Pakistan. This mucilaginous substance has strong adhesion as well as good emulsifying and binding properties. The Cordia gum has pseudoplastic behavior and high viscosity.

\section{Decontamination of gum:}

Sample was washed with double distilled water in order to expel dust and other surface impurities and then dried. Physical wash and spotlessness of gum was proceeded by following the procedure of Munir et al. ${ }^{[7]}$ with some modifications. Gum was soaked overnight in deionized water. A clingy thick arrangement/solution was gotten that was separated using a nankeen material (muslin cloth). This sifted arrangement/solution was introduced to $70 \%$ ethanol which resulted in smoggy white precipitates. The obtained precipitates were dried in oven at about $40-45^{\circ[8]}$.

\section{Modification of gum:}

By grafting: The purified gum sample (i.e. $1.00 \mathrm{~g}$ ) was dissolved in $25 \mathrm{ml}$ of distilled water. To the solution, acrylamide $\left(16 \times 10^{-2} \mathrm{M}\right)$, silver nitrate $\left(\mathrm{AgNO}_{3}\right)$ $\left(8 . \times 10^{-5} \mathrm{M}\right)$ and ascorbic acid $\left(22 \times 10^{-3} \mathrm{M}\right)$ were added. The solution was regulated in a water bath at $35^{\circ}$. After 30 min, potassium persulfate $\left(\mathrm{K}_{2} \mathrm{~S}_{2} \mathrm{O}_{8}\right)\left(8.0 \times 10^{-3} \mathrm{M}\right)$ was added and reaction was allowed for $1 \mathrm{~h}$. Ethanol was used to separate the modified gum from mixture. The precipitates (modified gum) were washed with ethanol and dried in hot air oven for further use $\mathrm{e}^{[9]}$.

By nanoparticles formulation: Pure gum was taken as green matrix to form $\mathrm{AgNps}$ following the method as reported by Munir et al. ${ }^{[7]}$. $\mathrm{AgNO}_{3}$ solution $(1 \mathrm{mM})$ was added drop wise in the solution of pure gum $(0.5 \% \mathrm{w} / \mathrm{v})$. This solution was then subjected to heat treatment at $121^{\circ}$ for $15 \mathrm{~min}$ that resulted in the evolution of AgNps. Nanoparticles were spectroscopically analyzed.

\section{Nano particles analysis by Zetasizer:}

Nanoparticles synthesized in the present research work were analyzed by particle size analyzer (Malvern zetasizer 2000, Malvern Instruments Ltd., U.K) at $24.9^{\circ}$ with $90^{\circ}$ detection angle ${ }^{[10]}$.

\section{Characterization of $C$. myxa samples:}

Ultraviolet/visible (UV/VIS) spectrophotometric analysis: Crude as well as nanoformulated samples of $C$. myxa were characterized by UV/Vis spectrophotometer (T60-UV-Visible/ Deuterium lamp/tungsten/halogen lamp). Detector used was the photodiode with scanning wavelength range of $190-1100 \mathrm{~nm}$. Resolution of spectra was $1 \mathrm{~nm}$ as reported by Kora et al. ${ }^{[11]}$.

\section{Biochemical analysis of crude gum:}

Proximate analysis: Proximate analysis (protein, moisture, ash, fats and fiber contents) of untreated gum was performed according to the protocol as reported in Association of Official Analytical Chemists (AOAC) ${ }^{[12]}$ and Galla and Dubasi ${ }^{[13]}$.

\section{Antioxidative potential:}

2,2-diphenyl-1-picryl-hydrazyl-hydrate (DPPH) radical scavenging activity: Potential of crude, 
purified as well as modified gum samples to scavenge free radicals was evaluated ${ }^{[14]}$.

Analysis of total phenolics: Total phenolics of samples under investigation were analyzed and the protocol with slight modification was adopted as reported by Ainsworth and Gillespie ${ }^{[15]}$.

Antimicrobial activity: Well diffusion method with different fungal strains was adopted for the evaluation of antiseptic behavior of experimental samples.

Antibacterial activity: Experimental samples of Cordia gum (i.e. crude, purified, acrylamide grafted and $\mathrm{AgNps}$ ) were subjected to antibacterial activity. Escherichia coli (E. coli) (ATCC 35218) and Staphylococcus aureus (S. aureus) (ATCC 25923) strains were used according to the method of Balouiri et al. ${ }^{[16]}$. Ampicillin was used as a positive control.

Antifungal activity: The activity of Cordia gum samples (i.e. crude, purified, acrylamide grafted and AgNps) to inhibit growth of Aspergillus niger (A. niger) and Fusarium solani (F. solani) was analyzed $^{[17]}$. Terbinafine was used as a positive control.

\section{Toxicological analysis:}

Assay for hemolysis: Hemolytic activity of experimental samples (i.e. crude, purified, acrylamide grafted and AgNps) was evaluated by running the sample according to the protocol reported by Irshad et al. ${ }^{[18]}$. Human blood samples $(3 \mathrm{ml}$ each) from volunteers were centrifuged $(850 \mathrm{xg})$ for $5 \mathrm{~min}$ and transferred to sterilized polystyrene tubes $(15 \mathrm{ml}$ capacity). Phosphate buffer saline (PBS) (pH 7.4) was used to isolate red blood cells (RBC) from plasma at $4^{\circ}$ and retained in $20 \mathrm{ml}$ chilled PBS. The diluted blood cell suspension $(180 \mu \mathrm{l})$ was added in erythrocyte cells (108 erythrocytes cells $/ \mathrm{ml}$ ) in a tube of $2 \mathrm{ml}$ capacity and incubated for $30 \mathrm{~min}$ at $37^{\circ}$ with agitation for $10 \mathrm{~min}$. Afterwards, kept in ice for $5 \mathrm{~min}$ and centrifuged at $1310 \mathrm{xg}$. The supernatant $(100 \mu \mathrm{l})$ was withdrawn from the tubes and diluted with $900 \mu \mathrm{l}$ chilled $\left(4^{\circ}\right)$ PBS.

All tubes were made stable on ice then introduced into 96 well plates $(200 \mu \mathrm{l})$. Triton X-100 $(0.1 \%)$ was taken as a positive control with maximum lysis whereas PBS was taken as a negative control with least lysis. Micro Quant ELISA plate reader (BioTek, Winooski, VT, USA) was used to measure intensity of absorption at $576 \mathrm{~nm}$.

The percentage lysis of RBCs was calculated by the following formula:
Lysis of RBCs (\%) =Absorbance of sample-Absorbance of negative control/Absorbance of positive control $\times 100$

Ames test for mutagenicity: For the evaluation of mutagenicity of the experimental samples, Ames test was performed using microbial strains such as Salmonella typhimurium (S. typhimurium) TA98 and $S$. typhimurium TA100. Nutrient agar was used to culture these bacteria at $4^{\circ} \pm 1^{\circ}$ and inoculation was done in nutrient broth and incubated at $37^{\circ}$ for $18-24 \mathrm{~h}$ prior to the analysis ${ }^{[19]}$.

Procedure used for Ames: Reagent mixture of DavisMingioli salt, Dextro-glucose, bromocresol purple, Dextro-biotin and Levo-histidine was prepared in a sterile bottle. Reagent mixture, gum, distilled water and standard mutagen were homogenized in number of bottles. Micro well plates were prepared according to the method of Razak and Aidoo $^{[20]}$ and sealed in plastic bags and incubated at $37^{\circ}$ for $4 \mathrm{~d}$. The reference plate was noted first followed by the experimental plates when all wells in blank were colored purple indicating the non-contaminated assay. The background, standard and test plates were scored visually. All yellow, partial yellow and turbid wells were counted as positive wells while purple wells were counted as negative. The extract was considered toxic to the test strain if all wells in the plate showed purple coloration. For an extract to be mutagenic, the number of positive wells had to be more than twice the number of positive wells in the background plate.

To perform Ames test, test sample $(0.005 \mathrm{ml})$ was run containing reagent mixture $(2.5 / 17.5 \mathrm{ml}$ deionized water) and Salmonella strain $(0.005 \mathrm{ml})$. Blank was run containing only reagent mixture whereas background contained reagent mixture along with microbial strain. Standard was run under the same conditions but without test sample.

\section{RESULTS AND DISCUSSION}

The selected gum was purified using ethanol precipitation method. The percentage of purified gum obtained and percent yield were estimated by comparing the gum used before and after the purification. A significant yield (i.e. $74.65 \%$ ) was obtained in the current research work (fig. 1a and fig. 1b).

Gum C. myxa was processed for biochemical analysis and results are given in Table 1. It is clear from the table that research sample contained contents of moisture $(9.68 \%)$, crude fats $(2.04 \%)$, ash $(2.58 \%)$, proteins 
$(2.49 \%)$, fibers $(2.21 \%)$ and carbohydrates $(88.41 \%)$ and total calculated energy was $349.84 \%$.

C. myxa as a green matrix was subjected to AgNps formulation that was confirmed by the development of dark yellow color (fig. 1c). Nano particles were further analyzed using UV/VIS spectrophotometry by running the crude and nanoformulated samples. Sharp peak at $428 \mathrm{~nm}$ was the confirmation of the formation of AgNps along with other smaller peaks in the region of $390-450 \mathrm{~nm}$ whereas in crude gum no sharp peak was present in this region but in the region of $423 \mathrm{~nm}$ (fig. 2).

In the samples under study, free radical scavenging activity was found maximum $(87.92 \%)$ shown by purified gum followed by polyacrylamide grafted gum $(85.86 \%)$, nanoformulated $(76.23 \%)$ and crude gum $(66.79 \%)$ as shown in Table 2.

Total phenolic contents were analyzed in the experimental samples and results are reported in Table 3. It is clear from the table that the crude sample contained phenolics up to $13.40 \pm 0.01 \mathrm{mg}$ whereas purified gum contained somewhat higher amount of phenolics $(14.71 \pm 0.03 \mathrm{mg})$. Minimum amount of phenolics was detected in grafted gum sample $(8.21 \pm 0.03 \mathrm{mg})$. Nano formulated gum sample also contained significant amount of phenolics $(10.73 \pm 0.03 \mathrm{mg})$.

Antibacterial activities of experimental samples were determined and results are given in Table 4. Antibacterial activity was found higher in nanoformulated sample. The calculated zones of inhibition against Bacillus subtilis (B. subtilus) and E. coli were $16 \mathrm{~mm}$ against both strains followed by crude (14 and $16 \mathrm{~mm}$ ), purified (14 mm each) and modified (12 mm each), respectively.

In the present research work, the antifungal activity was also evaluated against $A$. niger and $F$. solani (Table 5).

\section{TABLE 1: PROXIMATE ANALYSIS OF CRUDE} GUM C. myxa

\begin{tabular}{lcc}
\hline S. No. & C. myxa gum & Results \\
\hline 1 & Crude fat & $2.04 \pm 0.05$ \\
2 & Moisture content & $9.68 \pm 0.08$ \\
3 & Crude protein & $2.49 \pm 0.01$ \\
4 & Ash content & $2.58 \pm 0.05$ \\
5 & Crude fiber & $2.21 \pm 0.06$ \\
6 & Total carbohydrates & $88.41 \pm 0.36$ \\
7 & Nitrogen free extract & $91.01 \pm 0.04$ \\
8 & Total energy (kcal/g) & $349.83 \pm 0.74$ \\
\hline
\end{tabular}

TABLE 2: ACTIVITY OF C. myxa TO SCAVENGE FREE RADICAL

\begin{tabular}{lcc}
\hline S. No. & Sample ID & DPPH scavenging \% \\
\hline 1 & Crude & $66.79 \pm 3.53$ \\
2 & Purified & $87.92 \pm 2.25$ \\
3 & Acrylamide grafting & $85.86 \pm 1.71$ \\
4 & AgNps & $76.23 \pm 1.48$ \\
\hline
\end{tabular}
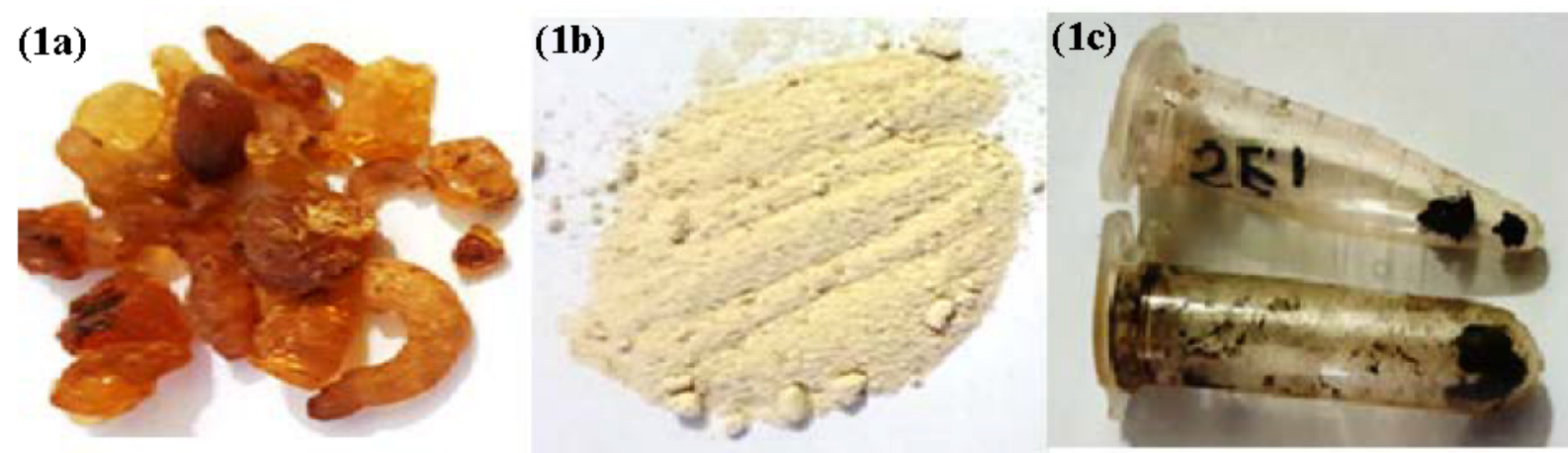

Fig. 1: (a) Crude gum; (b) Purified gum; (c) Nanoformulated gum
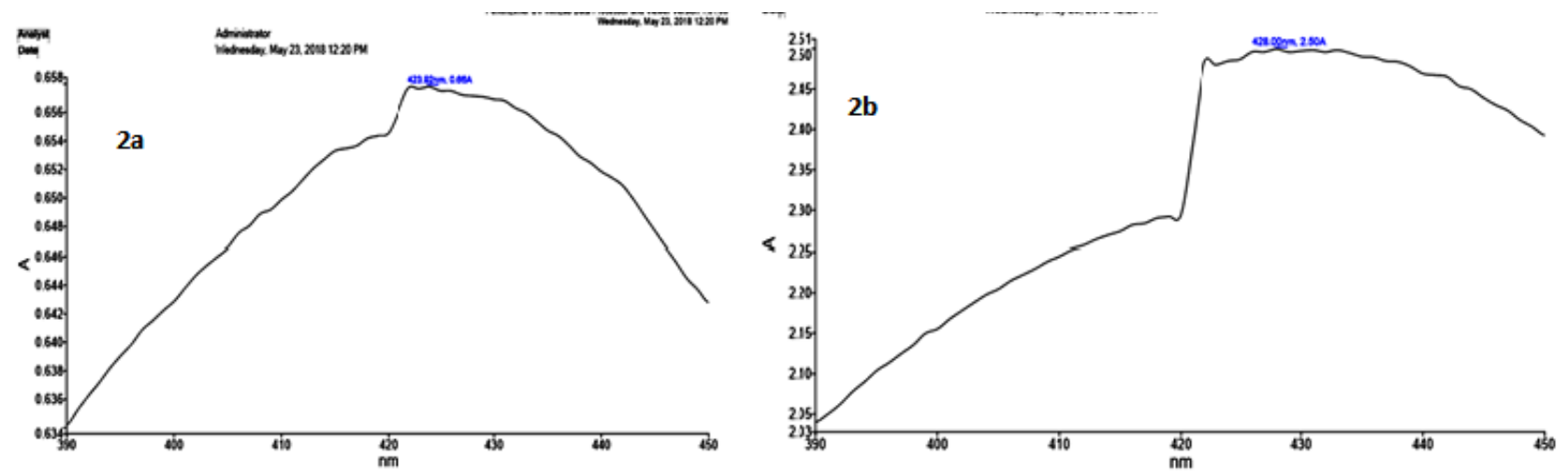

Fig. 2: (a) UV/Visible spectra of crude $C$. myxa; (b) UV/Visible spectra of nanoformulated $C$. myxa 
The crude gum sample as well as purified, modified and grafted samples exhibited excellent antifungal behavior. Maximum antifungal activity was demonstrated by the grafted sample and zone of inhibition was found to be $10 \mathrm{~mm}$ for $A$. niger and $F$. solani whereas minimum zone of inhibition of $5 \mathrm{~mm}$ against both strains was calculated of crude sample. Nano formulated and purified samples also showed significant values of antifungal activity and calculated zones of inhibition were 9.0 against $A$. niger and $11 \mathrm{~mm}$ against $F$. solani and $7 \mathrm{~mm}$ against both strains, respectively.

Experimental samples under investigation were subjected to hemolysis by taking human blood sample to examine toxicity (Table 6). Non-significant toxicity was observed by all the experimental samples. Toxicity of crude gum sample detected was found to be $8.19 \%$ and those of purified, modified and nanoformulated samples were found to be $8.53 \%, 6.01 \%$ and $9.91 \%$, respectively.

Mutagenicity of all experimental samples was analyzed by taking two bacterial strains $S$. typhimurium strain TA98 and S. typhimurium strain TA100 for the possible mutagenic activity and results are demonstrated in Table 7. It is clear from the table that all the experimental samples were non-mutagenic and not contaminated with mutagenic agents.

Ethanol was used as a solvent for the purification of selected gum. A change in color from brown to yellowish brown (due to removal of impurities) was detected in purified gum with the percentage yield of $74.65 \%$ (fig. 1a and fig. 1b). This purified gum might be utilized as additive in food products. The present findings were in accordance to the results reported by

TABLE 3: VALUES OF TOTAL PHENOLICS IN C. myxa GUM SAMPLES

\begin{tabular}{lcc}
\hline S. No. & Sample ID & $\begin{array}{c}\text { Total phenolic } \\
\text { (mg GAE/100 g dw) }\end{array}$ \\
\hline 1 & Crude & $13.4 \pm 0.01$ \\
2 & Purified & $14.71 \pm 0.03$ \\
3 & Acrylamide grafting & $8.21 \pm 0.03$ \\
4 & AgNps & $10.73 \pm 0.03$ \\
\hline
\end{tabular}

TABLE 4: ANTIBACTERIAL ACTIVITY OF C. myxa GUM SAMPLES AGAINST B. subtilus AND E. coli

\begin{tabular}{lccc}
\hline S. No & Sample ID & $\begin{array}{c}\text { B. subtilus } \\
(\mathbf{m m})\end{array}$ & E. coli $(\mathrm{mm})$ \\
\hline 1 & Crude & 14 & 16 \\
2 & Purified & 14 & 14 \\
3 & Acrylamide grafting & 12 & 12 \\
4 & AgNps & 16 & 16 \\
5 & Positive control & 20 & 24 \\
\hline
\end{tabular}

TABLE 5: ANTIFUNGAL ACTIVITY OF C. myxa AGAINST F. solani AND A. niger

\begin{tabular}{lccc}
\hline S. No. & Sample ID & F. solani & A. niger \\
\hline 1 & Crude & $5 \mathrm{~mm}$ & $5 \mathrm{~mm}$ \\
2 & Purified & $7 \mathrm{~mm}$ & $7 \mathrm{~mm}$ \\
3 & Acrylamide grafting & $10 \mathrm{~mm}$ & $10 \mathrm{~mm}$ \\
4 & AgNps & $11 \mathrm{~mm}$ & $9 \mathrm{~mm}$ \\
5 & Positive control (Terbinafine) & $20 \mathrm{~mm}$ & $15 \mathrm{~mm}$ \\
\hline
\end{tabular}

TABLE 6: HEMOLYTIC ACTIVITY OF C. myxa SAMPLES

\begin{tabular}{lcc}
\hline S. No. & Sample ID & Hemolysis \% \\
\hline 1 & Crude & $8.19 \pm 0.12$ \\
2 & Purified & $8.53 \pm 0.05$ \\
3 & Acrylamide grafting & $6.01 \pm 0.07$ \\
4 & AgNps & $9.91 \pm 0.10$ \\
5 & Positive control & $68.94 \%$ \\
\hline
\end{tabular}

Vinod and Sashidar ${ }^{[21]}$ and Kwakye et al. ${ }^{[22]}$. Vidyasagar et al. ${ }^{[23]}$ conducted their study to isolate and purify the C. dichotroma gum and their results are also in accordance with the results of current study.

After biochemical analysis, it is clear that this gum might be a rich source of basic nutrients such as proteins, carbohydrates, minerals, fibers and fats (Table 1). Values of these nutrients are somewhat higher as reported by other scientists in other gums. Murwan et al. ${ }^{[24]}$ performed proximate analysis of guar gum endosperms and detected moisture (4.8-8.7\%), protein (3.5-5.0\%), ash (0.5-0.8\%), fiber $(1.4-2.0 \%)$ and carbohydrate $(83.3-87.5 \%)$ whereas Gupta et al. ${ }^{[25]}$ reported contents of moisture, protein, fats and ash in the range of (7.4-12.4\%), (0.5\%-0.9\%), (0.29-0.34\%) and (0.80-0.86\%), respectively in three samples of guar gum obtained from different resources. Similarly, Osman et al. ${ }^{[26]}$ analyzed fiber contents $(2.3 \%)$ in guar gum. In consistent to our results, Jamil et al. ${ }^{[27]}$ have made the profile of proximate and mineral composition of uncharted date palm varieties collected from different geographical regions of Pakistan. According to them, moisture contents were 1.6 to $9.8 \%$ whereas soluble minerals were $1.82-2.87 \%$. They reported highly significant contents of proteins (32.5-41.25\%) as compared to results of present study and crude fiber was noticed in the range of 62.11 to $86.08 \%$ that is significantly lower as compared to present findings. It is assumed that gums and mucilages have higher amounts of crude fibers than seeds or fruits. These findings are in close agreement with the present research outcomes.

In AgNps formulation in current study, the gum was found to be expanded to have more surface area 


\begin{tabular}{lccccc}
\hline & \multirow{2}{*}{ S. No. } & $\begin{array}{c}\text { Sample } \\
\text { Name }\end{array}$ & \multicolumn{2}{c}{$\begin{array}{c}\text { S. typhimurium TA98 } \\
\text { No. of positive wells/total } \\
\text { wells }\end{array}$} & $\begin{array}{c}\text { Results } \\
\text { Ro. of positive wells/ } \\
\text { total wells }\end{array}$ \\
\hline 1 & Blank & - & Not contaminated & - & $\begin{array}{c}\text { Not } \\
\text { Results }\end{array}$ \\
2 & Background & $10 / 96$ & - & $10 / 96$ & contaminated \\
3 & Standard & $72 / 96$ & Mutagenic & $72 / 96$ & Mutagenic \\
4 & Crude & $1 / 96$ & Non-mutagenic & $2 / 96$ & Non-mutagenic \\
5 & Purified & $2 / 96$ & Non-mutagenic & $1 / 96$ & Non-mutagenic \\
6 & Grafted & $3 / 96$ & Non-mutagenic & $6 / 96$ & Non-mutagenic \\
7 & AgNps & $2 / 96$ & Non-mutagenic & $2 / 96$ & Non-mutagenic \\
\hline
\end{tabular}

for silver ions to react with functional group present on the gum matrix. The hydroxyl groups oxidized to carbonyl groups caused by the presence of silver ions thus resulted in the production of reduced elemental silver ${ }^{[28]}$ that were confirmed by the development of dark yellow color (fig. 1c). UV/VIS spectrophotometric analysis confirmed the formation of AgNps along with other smaller peaks in the region of $390-450 \mathrm{~nm}$ due to Surface plasmon resonance (SPR) whereas in the crude gum no sharp peak was present in this region but found at $423 \mathrm{~nm}$ (fig. 1b).

In purified as well as modified gum samples, optimal values of free radical scavenging activity were detected (Table 2). It is assumed that activity of flavoniods might be modified by the introduction of polyacrylamide that enhanced the free radical scavenging activity. Results of the current study are in accordance with previously reported results. $\mathrm{Xu}$ et al. ${ }^{[29]}$ modified the polysaccharides extracted from Ganoderma lucidum that resulted in the drastic boost in antioxidant behavior. These results are in strong agreement with the outcomes of Haq et al. ${ }^{[30]}$ who investigated gum C. myxa for extraction and characterization. These modified polymers exhibited excellent hydroxyl free radical scavenging activity $(83.4 \%)$ while the simple polysaccharide showed hydroxyl scavenging activity up to $42.9 \%$. Similarly these polymers also have the maximum potential to scavenge superoxide anion and hydrogen peroxide in comparison to the native polymer. The structure of the polysaccharide gets modified by the incorporation of carboxyl group that could be the cause of scavenging of free radicals. Munir et al. ${ }^{[7]}$ also studied DPPH scavenging effect of Dalbergia sissoo and Acacia modesta. They reported that crude gum contains high DPPH scavenging activity as compared to modified forms. The percentage scavenging effect was decreased in the hydrolyzed and modified samples as compared to the crude gum. This pattern of decreasing DPPH activity was shown by both selected gums.
Number of metabolites of phenolics and derivatives of phenolics are present in plants. These phenolic compounds have the ability to scavenge reactive oxygen species without causing further oxidative reactions ${ }^{[15]}$. In present research work, significant amount of phenolics were detected in the purified and modified gum samples (Table 3). In contrast to our results, Saleh et al. ${ }^{[31]}$ reported significantly higher amount of total phenolics in fruits of khalas variety of date palm (i.e. $238.54 \mathrm{mg} / 100 \mathrm{~g}$ dry weight (DW)). Louaileche et al. ${ }^{[32]}$ also detected phenolics (169.18 to $381.76 \mathrm{mg}$ gallic acid equivalent (GAE)/100 g) in aqueous extract of date fruits. These studies reported higher amounts of phenolics that could be due to the difference in varieties collected from different geographical regions. Munir et al. ${ }^{[7]}$ concluded that Acacia modesta exhibits more antioxidant activity. Crude gum samples were rich in phenolic compounds. But the amount of phenolics was decreased after purification, hydrolysis and modification. The consumption of these gums can be useful in degenerative diseases by preventing the oxidative stress. The analyzed gums can also be used as natural antioxidant in pharmaceutical and cosmetic industry.

Antibacterial activity was found higher in nanoformulated gum sample followed by crude, purified and modified gum samples (Table 4). Antibacterial activity of gum was affected by different steps of purification and modification. Crude gum sample exhibited maximum antiseptic behavior as it was not treated by any of the chemical reagents. One of the main purposes of modification given in literature is to minimize the microbial contamination possessed by crude gum and to increase their food and pharmaceutical applications. As the gum was subjected to purification as well as alteration, some of the functional groups that were responsible for antiseptic behavior might be lost and resulted in the change in the decrease in the antimicrobial behavior of the gum. According to the 
result of conducted antimicrobial assay the activity shown by modified and hydrolyzed samples was lower as compared to the crude gums. Similarly, Ravishanker and Raut et al. ${ }^{[33]}$ reported that date palm bark methanol fraction exhibited excellent activity against microbial strains with zones of inhibition of $22 \mathrm{~mm}$ against $S$. aureus and $20 \mathrm{~mm}$ against E. coli. Jayaprakash et $a .^{[34]}$ examined outstanding bactericidal effects of AgNps of fruit extract against eight bacterial strains. Fruit extract also showed excellent activity against microbes due to the presence of polyphenols.

In the present research work, the antifungal activity of crude gum sample as well as purified, modified and grafted samples exhibited excellent antifungal behavior. Maximum antifungal activity was demonstrated by the grafted gum sample and minimum was found by the crude gum sample. Nano formulated and purified samples also showed significant values of antifungal activities. Similarly, Boulenouar et al. ${ }^{[35]}$ assessed the antifungal potential of different date palm cultivars extracts against $F$. oxysporum and calculated zone of inhibition that was found to be $6.50 \mathrm{~mm}$.

Toxicity assay was performed to evaluate that modification in gum samples are beneficial or not (Table 6). It is clear from the table that non-significant toxicity was observed by all the experimental samples and values are in normal limits and therefore could be used as additives in nutraceuticals. In a study, Shahid et al. ${ }^{[36]}$ reported a little toxicity of grafted guar gum sample as compared to unprocessed gum. Similarly, Munir et $a .^{\left[{ }^{[7]}\right.}$ found no toxicity of aqueous extract of gums to human erythrocytes. Dhiman et al. ${ }^{[37]}$ reported that the grafted $C$. myxa gum has enhanced mucoadhesion and hence the grafted Cordia gum would be a promising excipient to develop mucoadhesive drug delivery systems.

To evaluate the DNA damaging activity of crude as well as modified gum samples, mutagenicity test was performed and results are depicted in Table 7. It is clear from the table that all the experimental samples were non-mutagenic and not contaminated with mutagenic agents. Das et al. ${ }^{[38]}$ also reported similar results of the samples under investigations.

In conclusion, the C. myxa gum is a medicinal gum and has been used in medicines as an excipient. The purification, modification and nanoformulation of Cordia gum were done in this study through green pathways. From present research findings it has been revealed that the purified gum has the highest free radical scavenging activity while nanoformulated gum has the highest antibacterial activity. Therefore, the modified gum has more nutritional as well as medicinal potential as compared to its conventional or crude form and has portrayed more benefits to food or other edible formulations as functional additives.

\section{Acknowledgements:}

The work was supported by a grant from Higher Education Commission, Islamabad, Government of Pakistan.

\section{Conflict of interests:}

The authors declare that there is no conflict of interests regarding the publication of this manuscript.

\section{REFERENCES}

1. Arif R, Ahmed S, Mustafa G. In silico study to reveal annotation and significant interactions of human defensin with its isoforms and their phylogeny. Indian J Pharm Sci 2020;82:465-71.

2. Al-Snafi AE. The Pharmacological and therapeutic importance of Cordia myxa-A review. IOSR J Pharm 2016;6(6):47-57.

3. Ahmed N, Khan MS, Jais AM, Mohtarrudin N, Ranjbar M, Amjad MS, et al. Anti-ulcer activity of sandalwood (Santalum album L.) stem hydro-alcoholic extract in three gastriculceration models of wistar rats. Bol latinoam Caribe plantas med aromát 2013;12(1):81-91.

4. Prajapati VD, Jani GK, Moradiya NG, Randeria NP, Nagar BJ. Locust bean gum: A versatile biopolymer. Carb Pol 2013;94(2):814-21.

5. Zhang XF, Liu ZG, Shen W, Gurunathan S. Silver nanoparticles: synthesis, characterization, properties, applications and therapeutic approaches. Int J Mol Sci 2016;17(9):1534.

6. Shah M, Fawcett D, Sharma S, Tripathy SK, Poinern GE. Green synthesis of metallic nanoparticles via biological entities. Materials 2015;8(11):7278-308.

7. Munir H, Shahid M, Anjum F, Akhtar MN, Badawy SM, ElGhorab. Application of Acacia modesta and Dalbergia sissoo gums as green matrix for silver nanoparticle binding. Green Process Synth 2016;5(1):101-6.

8. Sinha VR, Al-Azaki AA, Kumar RV. Novel Lannea Woodier gum matrices for controlled release of drugs. Carb Pol 2011;83(4):1492-8.

9. Singh V, Srivastava M, Tiwari A. Structural elucidation, modification and characterization of seed gum from Cassia javahikai seeds: A non-traditional source of industrial gums. Int J Biol Macromol 2009;45(3):293-7.

10. Jyoti K, Baunthiyal M, Singh A. Characterization of silver nanoparticles synthesized using Urtica dioica Linn. leaves and their synergistic effects with antibiotics. J Radiat Res Appl Sci 2016;9(3):217-27.

11. Kora AJ, Manjusha R, Arunachalam J. Superior bactericidal activity of SDS capped silver nanoparticles: synthesis and characterization. Mater Sci Eng: C 2009;29(7):2104-09.

12. AOAC. Official methods of analysis of Association of Official Analytical Chemists (AOAC). J AOAC Int, 18th ed 2011.

13. Galla NR, Dubasi GR. Chemical and functional characterization of Gum karaya (Sterculia urens L.) seed meal. Food Hydrocoll 2010;24(5):479-85.

14. Bozin B, Mimica-Dukic N, Simin N, Anackov G. 
Characterization of the volatile composition of essential oils of some Lamiaceae spices and the antimicrobial and antioxidant activities of the entire oils. J Agric Food Chem 2006;54(5):1822-28.

15. Ainsworth E, Gillespie KM. Estimation of total phenolic content and other oxidation substrates in plant tissues using Folin-Ciocalteu reagent. Nat Protoc 2007;2(4):875-7.

16. Balouiri M, Sadiki M, Ibnsouda SK. Methods for in vitro evaluating antimicrobial activity: A review. J Pharm Anal 2016;6(2):71-9.

17. Tariq M, Ali S, Muhammad N, Shah NA, Sirajuddin M, Tahir $\mathrm{MN}$, et al. Biological screening, DNA interaction studies and catalytic activity of organotin (IV) 2-(4-ethylbenzylidene) butanoic acid derivatives: synthesis, spectroscopic characterization and X-ray structure. J Coord Chem 2014;67(2):323-40.

18. Irshad M, Jones RC, Ahmed MN, Shahid M, Aziz S. New Pyrroloimidazoles by 1, 3-Dipolar Cycloaddition and their Biological Activities. J Chem Soc Pak 2017;39(1):113-18.

19. Ames BN, McCann J, Yamasaki E. Methods for detecting carcinogens and mutagens with the Salmonella/mammalianmicrosome mutagenicity test. Mutat Res 1975;31:347-63.

20. Razak MFA, Aidoo KE. Toxicity studies of Eurycoma longifolia (Jack)-Based remedial products. Asian J Pharm Clin Res 2011;4(3):1256-67.

21. Vinod VTP, Sashidhar RB. Surface morphology, chemical and structural assignment of gum Kondagogu (Cochlospermum gossypium DC.): an exudate tree gum of India. Natural Prod Rad 2010;1:181-92.

22. Kwakye G, Pronovost PJ, Makary MA. Commentary: a call to go green in health care by reprocessing medical equipment. Acad Med 2010;85(3):398-400.

23. Vidyasagar G, Jadhav AG, Narkhede SP, Narkhede SB. Isolation and comparative evaluation of Cordia dichotoma Forst. Mucilage as a binding agent with standard binder. J Chem Pharm Res 2010;2(4):722-6.

24. Murwan S, Abdelwahab A, Sulafa N. Quality Assessment of Guar Gum (Endosperm) of Guar (Cyamopsis tetragonoloba). ISCA J Biol Sci 2012;1:67-70.

25. Gupta S, Shah B, Sanyal B, Variyar PS, Sharma A. Role of initial apparent viscosity and moisture content on post irradiation rheological properties of guar gum. Food Hydrocolloids 2009;23(7):1785-91.

26. Osman NM, Amro BH, Mohamed-Ahmed IA, Babiker EE. Effect of autoclaving on solubility and functional properties of chickpea (Cicer arietinum) flour as a function of salt concentration. J Food Technol 2005;3(3):336-41.
27. Jamil MS, Nadeem R, Hanif MA, Ali MA, Akhtar K. Proximate composition and mineral profile of eight different unstudied date (Phoenix dactylifera L.) varieties from Pakistan. Afr J Biotechnol 2010;9(22):3252-9.

28. Vigneshwaran N, Ashtaputre NM, Varadarajan PV, Nachane RP, Paralikar KM, Balasubramanya RH. Biological synthesis of silver nanoparticles using the fungus Aspergillus flavus. Mater Lett 2007;61(6):1413-8.

29. Xu J, Liu W, Yao W, Pang X, Yin D, Gao Y. Carboxymethylation of a polysaccharide extracted from Ganoderma lucidum enhances its antioxidant activities in vitro. Carb Pol 2009; 78(2):227-34.

30. Haq, MA, Abid H, Khalid J, Haider MS. Extraction and characterization of gum from Cordia myxa. Asian J Chem 2014;26(1):122-6.

31. Saleh EA, Tawfik MS, Abu-Tarboush HM. Phenolic contents and antioxidant activity of various date palm (Phoenix dactylifera L.) fruits from Saudi Arabia. Food Nut Sci 2011;2:1134.

32. Louaileche H, Hammiche D, Hamoudi F. Total phenolic, flavonoid contents and in vitro antioxidant activity of Algerian date palm varieties: a comparative study. Am J Food Sci Health 2015;1(3):63-8.

33. Ravishanker R, Raut SV. Studies on antibacterial compounds from methanolic extract of bark of Phoenix dactylifera and its applications. Int J Curr Res 2016;8:28068-78.

34. Jayaprakash N, Vijaya JJ, Kennedy LJ. Microwaveassisted rapid facile synthesis, characterization, and their antibacterial activity of pvp capped silver nanospheres. Synth React Inorg Met Org Nano Met Chem 2015;45(10) :1533-38.

35. Boulenouar N, Marouf A, Cheriti A. Antifungal activity and phytochemical screening of extracts from Phoenix dactylifera L. cultivars. Nat Prod Res 2011;25(20):1999-02.

36. Shahid M, Bukhari SA, Gul Y, Munir H, Anjum F, Zuber $\mathrm{M}$, et al. Graft polymerization of guar gum with acryl amide irradiated by microwaves for colonic drug delivery. Int J Biol Macromol 2013;62:172-79.

37. Dhiman S, Asthana A, Tiwary AK, Jindal M, Shilakari G. Cordia myxa polysaccharide-G-poly(acrylonitrile) co-polymer based mucoadhesive gastroretentive delivery of captopril. Int J Pharm Sci Res 2016;7(1):259-71.

38. Das D, Ara T, Dutta S, Mukherjee A. New water resistant biomaterial biocide film based on guar gum. Bioresour Technol 2011;102(10):5878-83. 